Communications in Physics, Vol.21, No. 1 (2011), pp. 83-88

\title{
PULSE RESHAPING BY THE TWO-PORT NONLINEAR FIBER MACH-ZEHNDER INTERFEROMETER
}

\author{
HO QUANG QUY \\ Institute for Applied Physics-NEWTECHPRO \\ NGUYEN VAN HOA \\ Hong Duc University \\ NGUYEN THI THANH TAM \\ Quang Nam University
}

\begin{abstract}
In this article, the reshaping of the series of sinuous pulses and Gaussian pulses with arbitrary amplitude of laser signal by two-port nonlinear fiber Mach-Zehnder interferometer are simulated. The output series of close to flat-form pulses of laser signal is obtained. The influence of the input intensity, length of the nonlinear fiber, and nonlinear coefficient of refractive index on the shape of output pulse is discussed.
\end{abstract}

\section{INTRODUCTION}

The optical bistability is a phenomena in which occur two stable output states in proportion to one input state when a laser pulse transmits through a nonlinear medium. The cause of that phenomena is the abrupt change of physical states of system when the physical conditions (the parameters) change in given limits. This is an important property which contributes to the creation of high-speed quantum computer system. There are many kinds of the optical bistable device (OBD), whose operation is based on different principles, but most of them operate basing on the principle of nonlinear interferometer [1-4]. The previous OBDs are used in active mediums such as gas, liquid, but their dimension is too big. With the advantages in nano-semiconductor technology and optical fiber, the dimension of them is reduced more and more to nano-scale $[5,6,7,8,9]$. In the previous work [10], the transmittance as well as the bistability of Two-port nonlinear fiber Mach-Zehnder interferometer (NFMZI) have been investigated and discussed.

In this article, we present the pulse reshaping, one of the uses of Two-port NFMZI, and the influence of some principle parameters on the shape of output pulses.

\section{THE OUTPUT-INPUT RELATION OF INTENSITIES}

Consider a Four-port nonlinear fiber Mach-Zehnder interferometer, which consists from one nonlinear fiber, one linear fiber and two linear couplers (Fig.1a). After replacing two linear couplers by nonlinear couplers and two output ports connects one with other by a linear fiber we have a Two-port nonlinear fiber Mach-Zehnder interferometer (Fig.1b). The main purpose of this proposal is to use only one laser source in operating of Two-port NFMZI replacing to use two laser sources: one for pumping and other for controlling in the 
classical Four-port nonlinear Mach-Zehnder interferometer. As shown in previous work [10], the equation describes the output-input relation of intensities of Two-port NFMZI is given by

$$
\begin{aligned}
& I_{\text {out }}=I_{\text {in }} \times\left\{\left[-\left(2 \eta_{2}-1\right)\left(1-\eta_{1}\right)-2 \sqrt{\eta_{1} \eta_{2}\left(1-\eta_{1}\right)\left(1-\eta_{2}\right)}\right]^{2}\right. \\
& +\left[\left(2 \eta_{2}-1\right) \eta_{1}-2 \sqrt{\eta_{1} \eta_{2}\left(1-\eta_{1}\right)\left(1-\eta_{2}\right)}\right]^{2}-2\left[\left(2 \eta_{2}-1\right)\left(1-\eta_{1}\right)+2 \sqrt{\eta_{1} \eta_{2}\left(1-\eta_{1}\right)\left(1-\eta_{2}\right)}\right] \\
& \times\left[\left(2 \eta_{2}-1\right) \eta_{1}-2 \sqrt{\eta_{1} \eta_{2}\left(1-\eta_{1}\right)\left(1-\eta_{2}\right)}\right] \\
& \left.\times \cos \left(\frac{2 \pi n_{2} L}{\lambda\left(1-\eta_{1}\right)}\left[I_{\text {out }}+I_{\text {in }} \times\left(\eta_{1}\left(1-\eta_{1}\right)+4 \eta_{1} \eta_{2}\left(1-\eta_{1}\right)\left(1-\eta_{2}\right)+\eta_{1}^{2}\left(2 \eta_{2}-1\right)^{2}\right)\right]+\Delta \varphi\right)\right\}
\end{aligned}
$$

where $I_{\text {in }}, I_{\text {out }}$ are the input and output intensities, respectively, $n_{2}$ is the nonlinear coefficient of refractive index of nonlinear fiber, $L$ is the length of nonlinear fiber, $\lambda$ is the wavelength of laser signal, $\eta_{1}, \eta_{2}$ are transmittances of two nonlinear couplers, respectively, $\Delta \varphi$ is the initial phase shift of the laser field at input face of fiber (at the port $P_{i n}$ ).

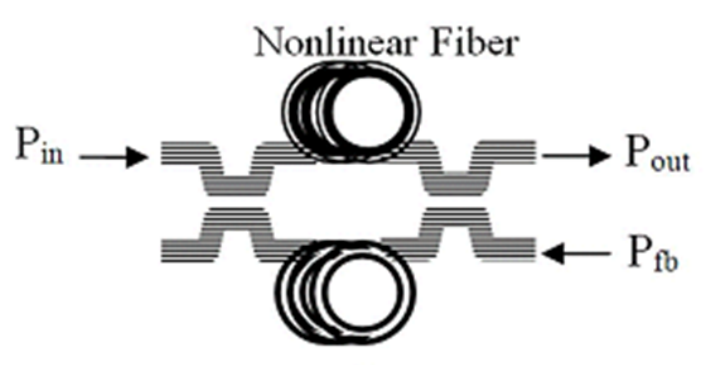

a

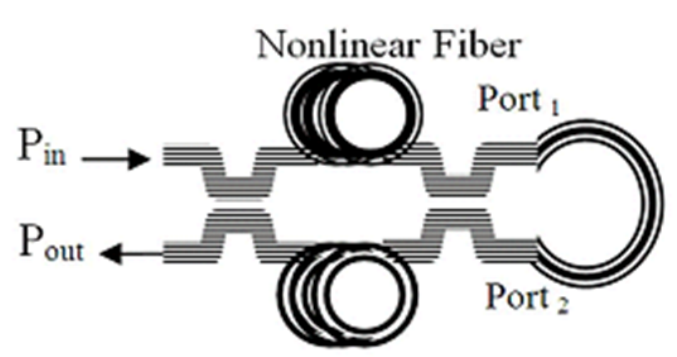

b

Fig. 1. (a) The Four-port NFMZI. (b) Two-port NFMZI.

This equation gives us to investigate the bistability of Two-port NFMZI, whose threshold intensity belongs to input intensity, nonlinear coefficient of refractive index, initial phase shift and length of nonlinear fiber. As well as known OBD, the Two-port NFMZI can be used for shaping the laser pulse, which will be investigated in detail in the next section.

\section{RESHAPING OF ARBITRARY PULSE OF LASER SIGNAL}

\section{III.1. Sinuous pulses with a constant amplitude}

Firstly, we consider the input laser signal is a series of sinuous pulses, whose shape is given by

$$
I_{\text {in }}=\frac{I_{\max }}{2}\left[1+\sin \left(\frac{-\pi(t+T)}{2 T}\right)\right],
$$

where $I_{\max }$ is the peak of intensity, i.e. intensity amplitude, which is chosen larger than intensity threshold of Two-port NFMZI, $T$ is the repeat period. Substituting (2) into (1) the full equation describes the input-output relation of intensities is rewritten as 


$$
\begin{aligned}
& I_{\text {out }}=\frac{I_{\max }}{2}\left[1+\sin \left(\frac{-\pi(t+T)}{2 T}\right)\right] \\
& \times\left\{\left[-\left(2 \eta_{2}-1\right)\left(1-\eta_{1}\right)-2 \sqrt{\eta_{1} \eta_{2}\left(1-\eta_{1}\right)\left(1-\eta_{2}\right)}\right]^{2}\right. \\
& +\left[\left(2 \eta_{2}-1\right) \eta_{1}-2 \sqrt{\eta_{1} \eta_{2}\left(1-\eta_{1}\right)\left(1-\eta_{2}\right)}\right]^{2}-2\left[\left(2 \eta_{2}-1\right)\left(1-\eta_{1}\right)+2 \sqrt{\eta_{1} \eta_{2}\left(1-\eta_{1}\right)\left(1-\eta_{2}\right)}\right] \\
& \times\left[\left(2 \eta_{2}-1\right) \eta_{1}-2 \sqrt{\eta_{1} \eta_{2}\left(1-\eta_{1}\right)\left(1-\eta_{2}\right)}\right] \\
& \times \cos \left(\frac { 2 \pi n _ { 2 } L } { \lambda ( 1 - \eta _ { 1 } ) } \left[I_{\text {out }}+\frac{I_{\max }}{2}\left[1+\sin \left(\frac{-\pi(t+T)}{2 T}\right)\right]\right.\right. \\
& \left.\left.\left.\quad \times\left(\eta_{1}\left(1-\eta_{1}\right)+4 \eta_{1} \eta_{2}\left(1-\eta_{1}\right)\left(1-\eta_{2}\right)+\eta_{1}^{2}\left(2 \eta_{2}-1\right)^{2}\right)\right]+\Delta \varphi\right)\right\}
\end{aligned}
$$

Numerically solving (3) by Mathematica software using typical parameters as: $\lambda=$ $1.33 \mu \mathrm{m}, n_{2}=1 \times 10^{-14} \mathrm{~cm}^{2} / W, L=100 \mathrm{~cm}, \Delta \varphi=-0.3 \pi, I_{\max }=0.3 \times 10^{8} \mathrm{~W} / \mathrm{cm}^{2}$, $\eta_{1}=\eta_{2}=0.5$, and $T$ chosen arbitrary, the input and output series of pulses are simulated and illustrated in Fig.2a.

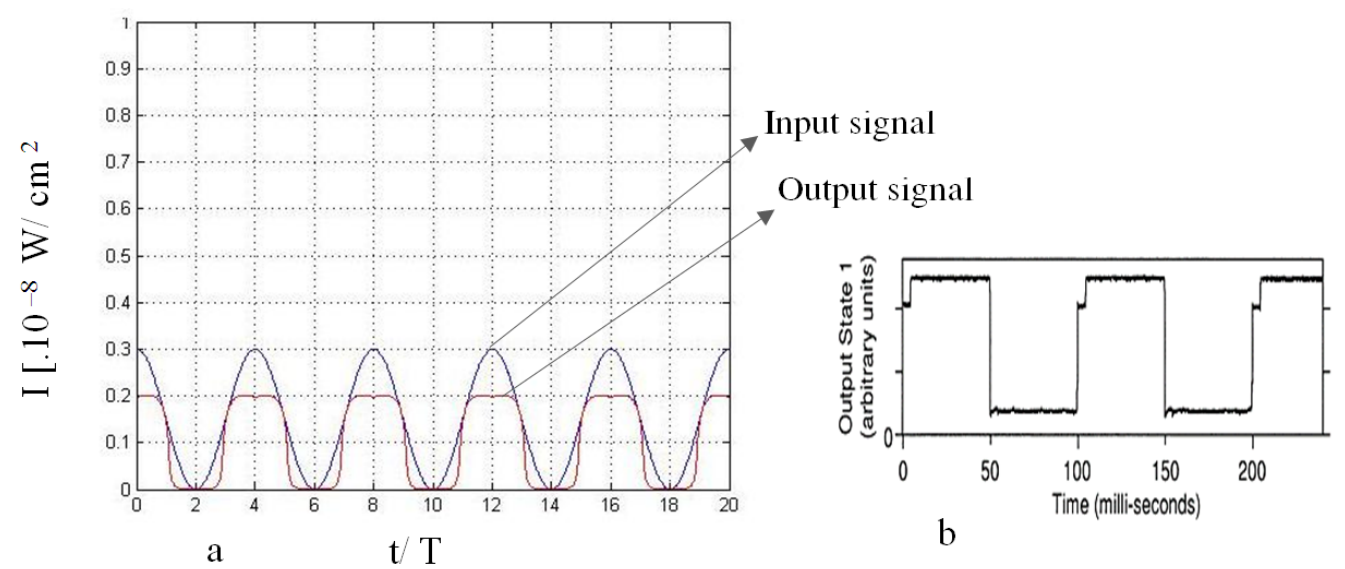

Fig. 2. The shape of input and output series of laser pulses. (a) Simulated results for Two-port NFMZI. (b) Optical flip-flop on classical Four-port NFMZI from work $[6]$.

From Fig.2a, we can see that the input sinuous pulse will be reshaped through Twoport NFMZI, and then become a rectangle "chain", whose pulse is closed to "flat-form" on top, where $I_{\text {out } \text { max }}=0.2 \times 10^{8} \mathrm{~W} / \mathrm{cm}^{2}$, and under deep, where $I_{\text {out } \text {,min }}=0$. This "chain" is absolutely logical to that received on work of Hill [6], in which two Four-port NFMZIs have been connected to design the flip-flop shape of output pulses (Fig.2b). 


\section{III.2. Sinuous pulse with arbitrary amplitude}

Now, we consider the input laser signal is a series of sinuous pulses with arbitrary amplitude. Its shape is given as follows

$$
I_{\text {in }}(t)=\frac{I_{\max }\left(1+m h(t) I_{m}\right)}{2}[1+\sin (2 \pi(t+T / 4))]
$$

where $I_{\max }$ is the intensity peak, i.e. a constant amplitude, which is chosen larger then intensity threshold of Two-port NFMZI, $T$ is the repeat period, $m$ is the modulating deepness, $h(t) I_{m}$ is the modulating amplitude, $h(t)$ is random function. Substituting (4) to (1) and considering $\eta_{1}=\eta_{2}=0.5$ for simplicity, we have

$$
\begin{aligned}
I_{\text {out }}= & \frac{I_{\max }\left(1+m h(t) I_{m}\right)}{2}[1+\sin (2 \pi(t+T / 4))] \\
& \times\left(\frac{1}{2}+\frac{1}{2} \cos \left(\frac{4 \pi n_{2} L}{\lambda}\left(\begin{array}{l}
I_{\text {out }}+\frac{1}{2} \frac{I_{\max }\left(1+m h(t) I_{m}\right)}{\left[1+\sin \left(2 \pi\left(t+T^{2} / 4\right)\right)\right.} \times
\end{array}\right)+\Delta \varphi\right)\right) .
\end{aligned}
$$

Numerically solving with $I_{\max }=2.0 \times 10^{9} \mathrm{~W} / \mathrm{cm}^{2}, I_{m}=0.5 \times 10^{8} \mathrm{~W} / \mathrm{cm}^{2}, \mathrm{~m}=8$, $T$ chosen arbitrary, the random function has value changing randomly from 0 to 1 , i.e. $h=0 \div 1$, and other parameters are given as above, the series of input pulses and the output pulses are simulated and illustrated in Fig.3.

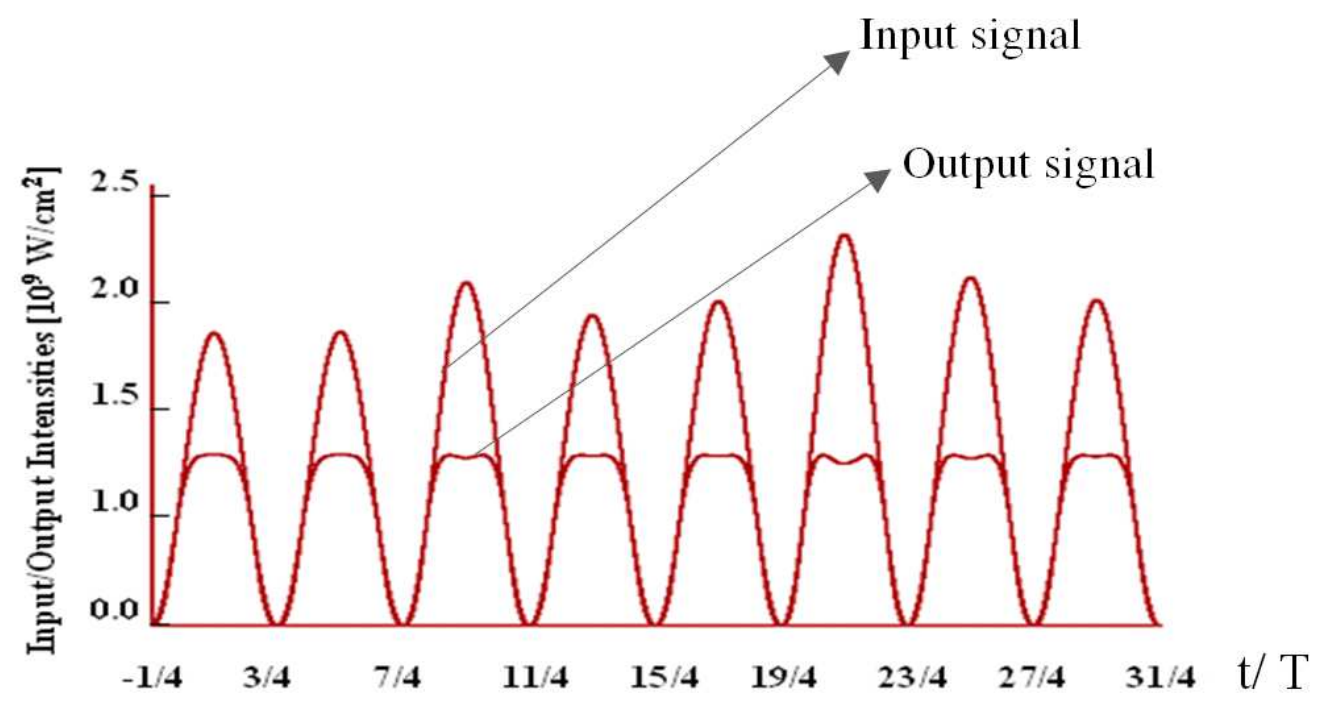

Fig. 3. The shape of input and output of sinuous pulses.

From Fig.3, we can see that, the input sinuous pulse will be shaped through Twoport NFMZI, and then become a rectangle "chain", whose pulse is closed to "flat-from" ones with equal peak of intensity, $I_{\text {out }, \text { max }} \approx 1.25 \times 10^{9} \mathrm{~W} / \mathrm{cm}^{2}$. 


\section{III.3. Series of Gaussian pulses with arbitrary amplitude}

In the unstable fact of laser operation, a series of closed to Gaussian pulses with arbitrary amplitude can be appeared. Mathematically, the dependence of laser intensity on time can be approximately expressed in the form as

$$
I_{i n}(t)=\left(I_{0}+m h(t) I_{m}\right) e^{-\left(\frac{\sqrt{\ln 2}(t+3 T)}{T}\right)^{2}},
$$

where $I_{0}$ is given constant amplitude, which is chosen to be lager than the threshold intensity of Two-port NFMZI, $h(t) I_{m}$ is the modulating amplitude, $h(t)$ is random function, $m$ is the modulating deepness, $T$ is the repeat period of the series. Substituting (2) to (1), the full equation describing the relation of intensities is given by

$$
\begin{aligned}
I_{\text {out }}= & \left(I_{0}+m h(t) I_{m}\right) e^{-\left(\frac{\sqrt{\ln 2}(t+3 T)}{T}\right)^{2}} \\
& \left.\left(\begin{array}{l}
\frac{1}{2}+\frac{1}{2} \cos \left(\frac{4 \pi n_{2} L}{\lambda}\left(\begin{array}{c}
I_{\text {out }}+\frac{1}{2}\left(I_{0}+m h(t) I_{m}\right) \times \\
e^{-\left(\frac{\sqrt{\ln 2}(t+3 T)}{T}\right)^{2}}
\end{array}\right)+\Delta \varphi\right.
\end{array}\right)\right),
\end{aligned}
$$

here, we consider $\eta_{1}=\eta_{2}=0.5$ for simplicity. Using principle parameters as: $I_{0}=$ $2 \times 10^{9} \mathrm{~W} / \mathrm{cm}^{2}, n_{2}=1 \times 10^{-14} \mathrm{~cm}^{2} / W, I_{m}=0.5 \times 10^{9} \mathrm{~W} / \mathrm{cm}^{2}, \lambda=1.33 \mu \mathrm{m}, L=100 \mathrm{~cm}$, the random function has value changing randomly from 0 to 1 , i.e. $h=0 \div 1$, and chosen arbitrary, the series of input and output pulses are illustrated in Fig.4a.

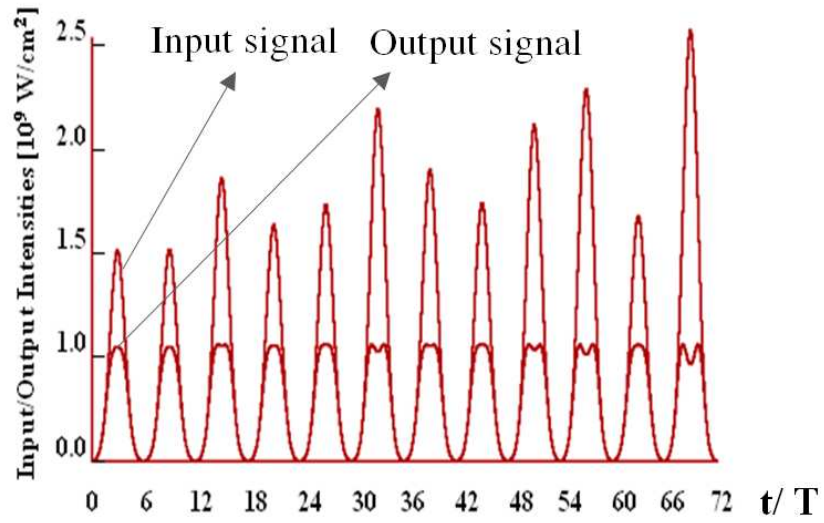

a

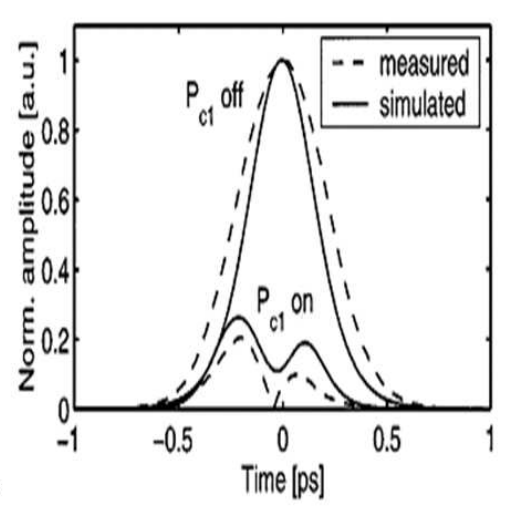

b

Fig. 4. The shape of input and output series of close to Gaussian laser pulses with arbitrary amplitude. (a) Simulated results for TPNFMZI. (b) Experimental and Theoretical results of MZI from work [11].

From this Fig.4a, it can be seen that though the peaks of input pulses (pulses with higher peak) change randomly causing by the random function $h(t)$, but the peaks of output pulses nearly not change with increasing of modulating deepness $m$. Moreover, it is clearly that most of output pulses is closed to "flat-form" ones with equal peak of intensity, $I_{\text {out }, \max } \approx 1.15 \times 10^{9} \mathrm{~W} / \mathrm{cm}^{2}$. 
The "flat-form" of the output pulse will be broken when the greater input intensity. Finally, the output pulse has a "hole" form, which is in good agreement to simulated and experimental results in Schreiech's and Krijnen works [11] (see Fig. 4b).

From the equations (1), (3), (5), (7), we can conclude that the shape of output pulse depends not only on the input intensity (or amplitude), but on the length and nonlinear coefficient of refractive index of nonlinear fiber, and initial phase shift.

\section{CONCLUSIONS}

By simulation, the obtained results show that the Two-port NFMZI will be used for reshaping the laser pulse. The series of arbitrary pulses can be reshaped to a series of closed to uniform pulses. The laser pulse shaping character of Two-port NFMZI leads to use it for the analog-to-digital conversion of laser signal and for optical communication, in which the unstable laser signal will be stabilized. Moreover, with mentioned above character the Two-port NFMZI can replace the NFMZI with four ports.

Our results have been simulated using only a given collection of parameters for ex-

ample, but it is fact that the shape of output pulse depends on principle parameters of Two-port NFMZI, as input intensity, duration and repeat period of pulse, nonlinear coefficient of refractive index, length of nonlinear fiber, initial phase shift, and transmittance of nonlinear coupler, whose is chosen constant $(3 \mathrm{~dB})$ in this article. These parameters not only influence the peak, but also influence the shape and duration of the output pulse. The remaining questions will be investigated in the next works.

\section{REFERENCES}

[1] M. C. D. Andrew, Opt. Switching, OPN, (2005) 34.

[2] L. Brzozowski et al., J. of Lightwave Technology 19 (2001) 114-119.

[3] N. V. Hoa, H. Q. Quy, V. N. Sau, Commun. in Phys 15 (2005) 223-228.

[4] N. V. Hoa, H. Q. Quy, D. X. Khoa, Proc. of GVS6 Chemnitz, (2003) 144-147.

[5] A. Erlacher et al, J. of Appl. Phys. 95 (2004) 2927-2929.

[6] M. T. Hill, Otp. Tech. Letter 31 (2001) 411-415.

[7] H. Q. Quy, V. N. Sau, N. V. Hoa, Comm. in Phys. 13 (2003) 157-164.

[8] H. Sakata, Appl. Phys. 40 (2001) 240-248.

[9] M. Soljacic et al, Phys. Rev. E66 (2002) 05560-1-05560-4(R).

[10] H. Q. Quy, V. N. Sau, N. T. T. Tam, and N. V. Hoa, Comm. in Phys. 20 (2010) 45-50.

[11] R. Schreiech, M. Kwakernaak, IEEE Photonics Technology Letters 13 (2001) 603-605.

Received 08 July 2010. 